\title{
Unrepeatered OTDM Data Transmission over Long Legacy Fiber Span Using Unidirectional Backward Raman Amplification
}

\author{
Mousaab M. Nahas \\ Electrical and Computer Engineering Department, Faculty of Engineering, University of Jeddah, Jeddah, Saudi Arabia \\ Email address: \\ mnahas1@uj.edu.sa

\section{To cite this article:} \\ Mousaab M. Nahas. Unrepeatered OTDM Data Transmission over Long Legacy Fiber Span Using Unidirectional Backward Raman \\ Amplification. Journal of Electrical and Electronic Engineering. Vol. 3, No. 5, 2015, pp. 127-132. doi: 10.11648/j.jeee.20150305.15
}

\begin{abstract}
This paper presents experimental results for transmitting $40 \mathrm{~Gb} / \mathrm{s}$ OTDM signal over in-line long fiber span using unidirectional backward Raman amplification. The investigation uses legacy dispersion-managed SMF-DCF configuration where remote Erbium amplification is used to compensate for the DCF spans losses. It is practically shown that the system performance improves significantly with more Raman pump power if we use an appropriate signal wavelength, Raman pump power and Erbium gain. As a result, successful unrepeatered transmission over $206 \mathrm{~km}$ SMF is achieved using $1545 \mathrm{~nm}$ signal wavelength, $1.58 \mathrm{~W}$ Raman power and unsaturated EDFA gains into the DCF spans. We believe that the results of such investigation can be useful for enhancing systems that still use legacy cables without the need for substantial alteration.
\end{abstract}

Keywords: Fiber-Optic Communications, OTDM, Raman Amplification

\section{Introduction}

It is well known in the optical telecommunications arena that Raman amplification is referred to the Stimulated Raman Scattering (SRS) phenomenon where signal amplification is attained via nonlinear power transfer from an intense pump beam propagating simultaneously through the fiber [1]. It is also traditionally recognized that Raman amplification can be applied in either forward or backward direction. In forward Raman pumping, the energy is transferred from the pump beam to the data signal as the two beams co-propagate inside the fiber. In backward Raman, the pump and data signal beams counter-propagate in the fiber, and this type is commonly used in practice due to better amplification results [2]. In reality, most of the long haul optical transmission systems $(\geq 200 \mathrm{~km}$ ) use Raman amplification since the early part of the $20^{\text {th }}$ century [3]. Moreover, plenty of researches have already demonstrated successful transmission using unrepeatered Raman amplification. Many of these researches use multiple $10 \mathrm{~Gb} / \mathrm{s}$ WDM signals [4-7], while many others use higher bit rates through OTDM as we demonstrate in this research. However, some of these OTDM projects use allRaman amplification that is split between the SMF and DCF spans, where no EDFA amplification is applied [8]. In other projects, unconventional large effective area fiber (LEAF) is used in which the nonlinear penalty is reduced including SRS, thus high power of Raman pump is required to increase amplification [9-11]. Other unconventional fibers are also used like DSF and NZ-DSF [12-13] that we are not interested in hereby. In later project [14], conventional SMF is used but the authors apply bidirectional Raman pumping scheme. Recently, some projects use conventional SMF in alldistributed Raman configuration [15-16] where multiple Raman modules are distributed around short or medium SMF spans so that transmission over unrepeatered long spans is not demonstrated.

In this paper, we investigate the application of unidirectional backward Raman amplification over unrepeatered long conventional SMF using $40 \mathrm{~Gb} / \mathrm{s}$ OTDM signal with $2^{31}-1$ data length. This signal is being encoded by a simple traditional coding scheme, which is RZ-IMDD, such that the complexity of the transmitter and receiver is minimized. This effectively opens the door for upgrading already installed systems or legacy parts of a network without the need for adding any complexity. However, there have been results of unidirectional Raman over long conventional fibers [17], but since the experiments are too old they use $2^{7}-1$ word length which is much shorter that what we have in this project. 


\section{Experimental Setup}

Fig. 1 shows the experimental setup for our investigation. An RF generator produces $10 \mathrm{GHz}$ electrical signal that is used to drive both a laser source and a pulse pattern generator (PPG). The PPG produces a PRBS data signal with $2^{31}-1$ length which is used to modulate the laser signal at a $\mathrm{LiNbO}_{3}$ intensity modulator. The output of this process is basically a $10 \mathrm{~Gb} / \mathrm{s}$ RZ-IM data signal. This resulting bit rate is experimentally increased to $40 \mathrm{~Gb} / \mathrm{s}$ through Mach Zehnder optical time division multiplexing (MZ-OTDM) that is shown in Fig. 2, where the output pulses produced by the laser mentioned above are sufficiently narrow. In MZ-
OTDM, a $10 \mathrm{~Gb} / \mathrm{s}$ signal whose pulse time window is $100 \mathrm{ps}$ is split into two channels; one is delayed by 50 ps and then couples back with the other channel. The resultant is $20 \mathrm{~Gb} / \mathrm{s}$ signal. If this stage is repeated with 25 ps delay, the output signal is then $40 \mathrm{~Gb} / \mathrm{s}$. In practice, such delays are insufficient for adequate mixing of bits as they would result in sequential bit repetition within the combined $40 \mathrm{~Gb} / \mathrm{s}$ random data. Therefore, we use additional 100 ps (one time widow) delay to avoid this effect [18]. Polarization controller is used in one Mach Zehnder arm to equalize the polarization of the two arms. The MZ-OTDM input $10 \mathrm{~Gb} / \mathrm{s}$ and output $40 \mathrm{~Gb} / \mathrm{s}$ signals are shown in Fig. 3.

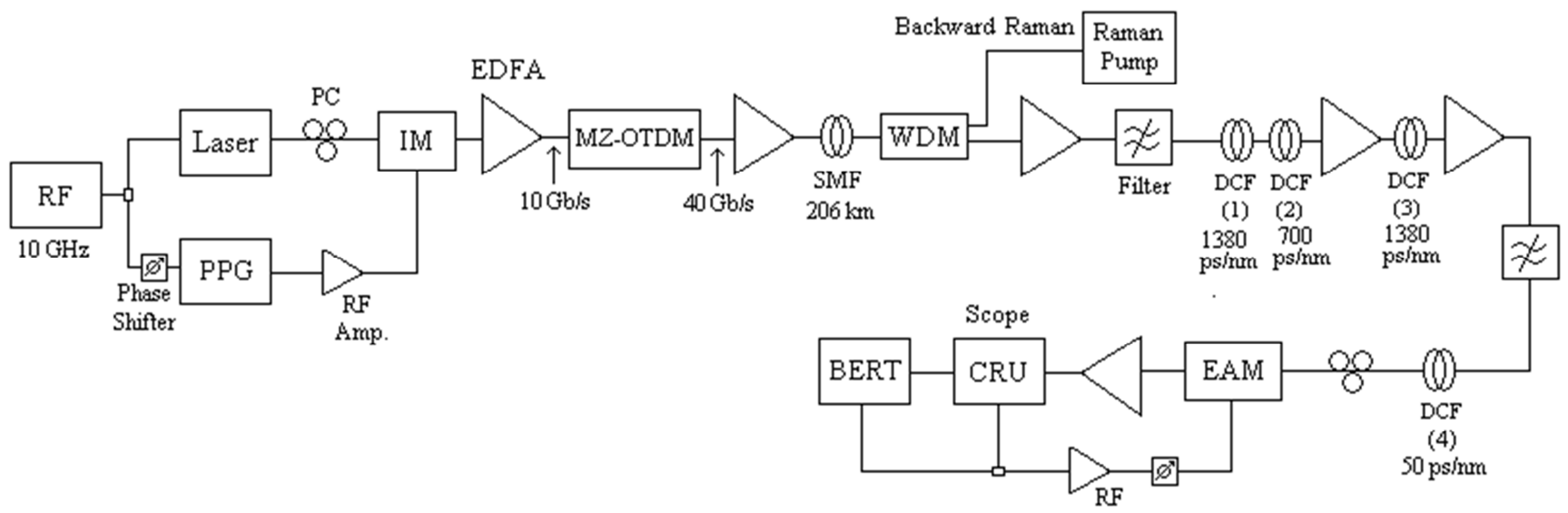

Figure 1. Experimental setup.

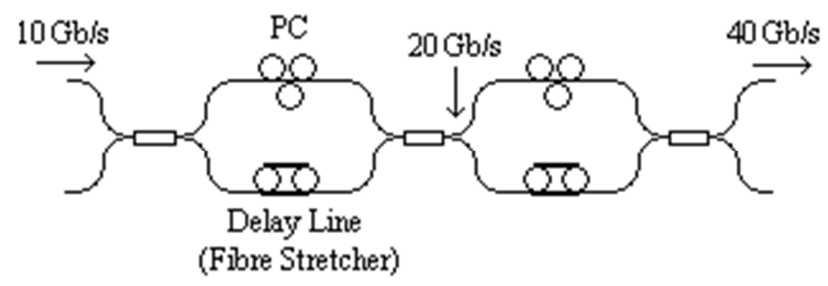

Figure 2. MZ-OTDM.

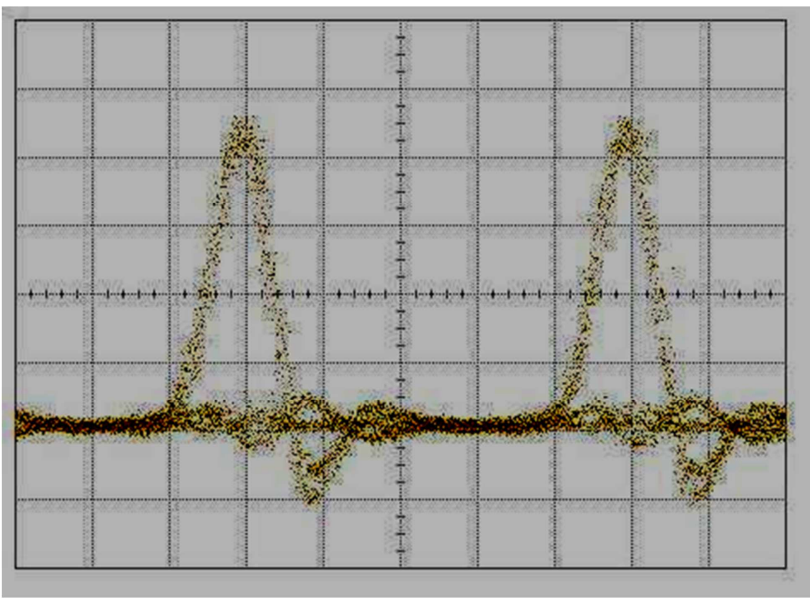

(a)

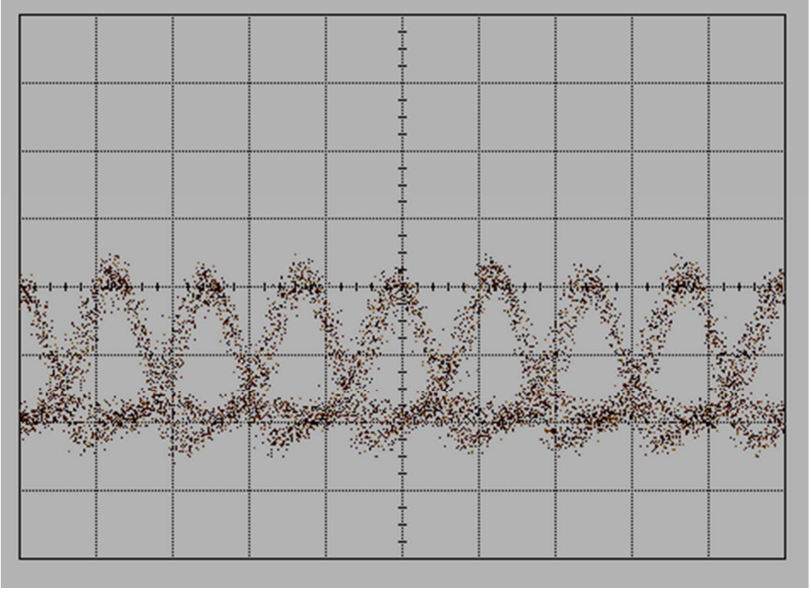

(b)

Figure 3. (a) MZ-OTDM input $10 \mathrm{~Gb} / \mathrm{s}$ signal; (b) MZ-OTDM output 40 $\mathrm{Gb} / \mathrm{s}$ signal.

The resulting $40 \mathrm{~Gb} / \mathrm{s}$ signal is boosted by an EDFA and then transmitted over a $206 \mathrm{~km}$ SMF span. The average fiber attenuation is measured to be $0.2 \mathrm{~dB} / \mathrm{km}$ at $1550 \mathrm{~nm}$, while the total dispersion is measured to be $3510 \mathrm{ps} / \mathrm{nm}$. This dispersion is compensated by the DCF modules shown in the setup. The loss of the SMF span is to be compensated by backward Raman as seen, while the cascaded EDFAs are used to compensate for losses in the DCF spans and in other components such as filters. The filters are used to eliminate 
the accumulative ASE noise along the system.

At the receiver, the individual $10 \mathrm{~Gb} / \mathrm{s}$ signal must be extracted from the $40 \mathrm{~Gb} / \mathrm{s}$ OTDM bit stream for measurements. Since each pulse occupies a 25 ps window in the $40 \mathrm{~Gb} / \mathrm{s}$ signal, it is required to create a $25 \mathrm{ps}$ switching window every 100 ps to extract a $10 \mathrm{~Gb} / \mathrm{s}$ channel. To achieve this, an electro-absorption modulator (EAM) is used to absorb the unwanted three channels and leave only one channel in the time window. The EAM is initially driven by a $10 \mathrm{GHz}$ electrical signal generated by clock recovery unit (CRU) to enable modulation of the first arrived bits, and then it is driven through feedback clock recovery for the next coming bits. The phase of the $10 \mathrm{GHz}$ signal can be adjusted using a phase shifter which enables sliding the switching window in the time domain, giving the ability to select which of the four OTDM channels to be detected. The output 10 $\mathrm{Gb} / \mathrm{s}$ signal is isolated and pre-amplified before being detected and analyzed.

\section{Results and Discussion}

Fig. 4 shows the back-to-back demultiplexed RZ data signal obtained from the above setup (without transmission) using $-1 \mathrm{dBm}$ EAM input power. It is obvious that the receiver demonstrated above can successfully extract a single $10 \mathrm{~Gb} / \mathrm{s}$ channel out of the entire OTDM signal, where the original pluses are recovered properly and no errors are counted at the BERT.

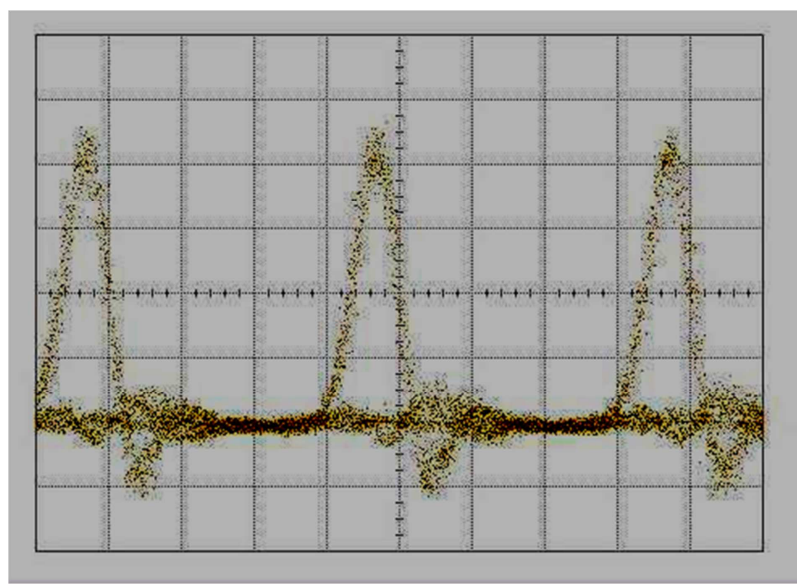

Figure 4. Back-to-back $10 \mathrm{~Gb} / \mathrm{s}$ signal.

In transmission over $206 \mathrm{~km}$, it is initially observed that the signal performance is varying with the wavelength, which is common with most laser sources that produce narrow pulses due to chirp variation. Therefore, it is significant to identify the best operating wavelength for this system before applying Raman amplification. Fig. 5 shows the signal BER as a function of wavelength using high launched power (17 $\mathrm{dBm}$ ) and EDFA amplification only. As a result, the best performance is found to be around $1545 \mathrm{~nm}$, thus this is the operating wavelength for our data signal from now on. Obviously, the BER is not aimed to be optimized at this stage as it is used here just for comparison purpose.

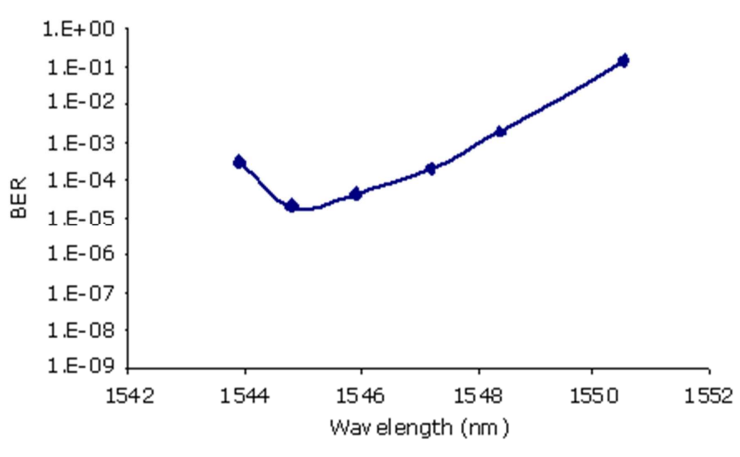

Figure 5. BER vs operating wavelength.

To apply Raman amplification, the Erbium gains are set to mainly compensate for the DCF losses which are $\sim 0.5$ $\mathrm{dB} / \mathrm{km}$ in all DCF spans. This Erbium gain should also cover the filters losses as stated before. However, the signal launched power used at this stage is chosen to be $13 \mathrm{dBm}$, and this is based on the existing EDFAs saturation levels. This would imply that all Erbium amplifiers are allowed to work near saturation. By applying backward Raman pump signal at $1455 \mathrm{~nm}$ to the $206 \mathrm{~km}$ SMF span, the received signal counts considerable number of errors and is significantly distorted. This can be understood from Fig.6 (a) that shows the output spectra from the first amplifier, i.e. after the SMF span, using different pump powers in the range $40-100 \%$ according to the existing pump module. The corresponding values of the percentages mentioned above (and seen in the figure) are 0.06, 0.353, 0.624, 0.894, 1.13, 1.37 and $1.58 \mathrm{~W}$, respectively. In (b), the OSNR measurements at the same point are presented versus Raman pump for higher and lower wavelengths. It is clear from both (a) and (b) that, the lower the Raman pump, the higher the ASE peak at $1530 \mathrm{~nm}$, while the higher the pump, the higher the ASE noise around $1555 \mathrm{~nm}$. However, the received BER against Raman pump is shown in Fig. 7. It is noticeable that the Raman pump increases the signal BER so far. Fig. 8 shows the eye closure due to increased Raman pump, where the worst eye is observed at $100 \%$ pump power. Practically, this degradation is mainly caused due to the interaction between Raman signal and the ASE noise signal caused by the lumped Erbium amplification, where this interaction is wavelength dependent.

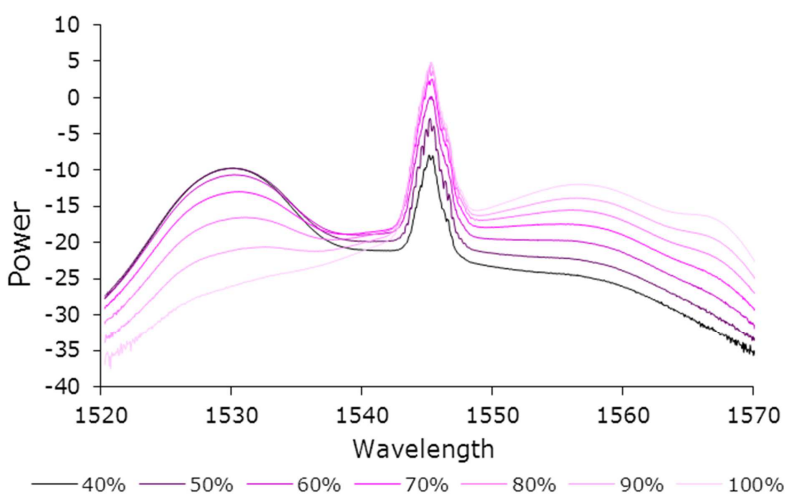

(a) 


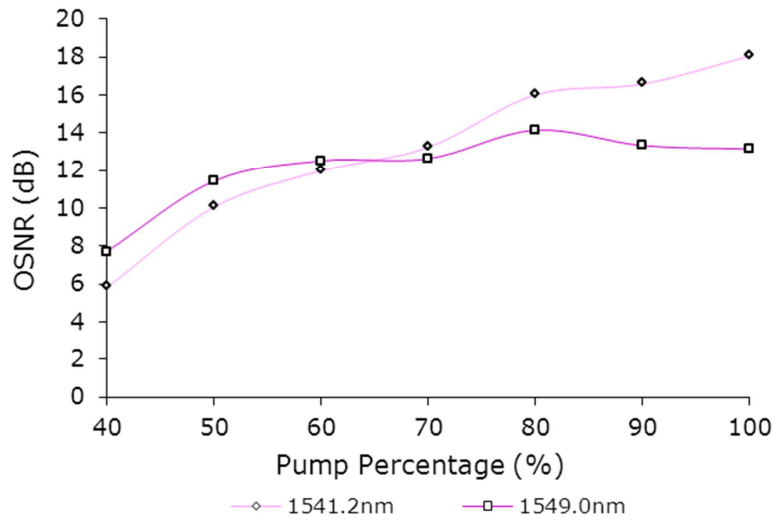

(b)

Figure 6. (a) SMF output spectra for different Raman pumps. (b) SMF output OSNR vs Raman pump.

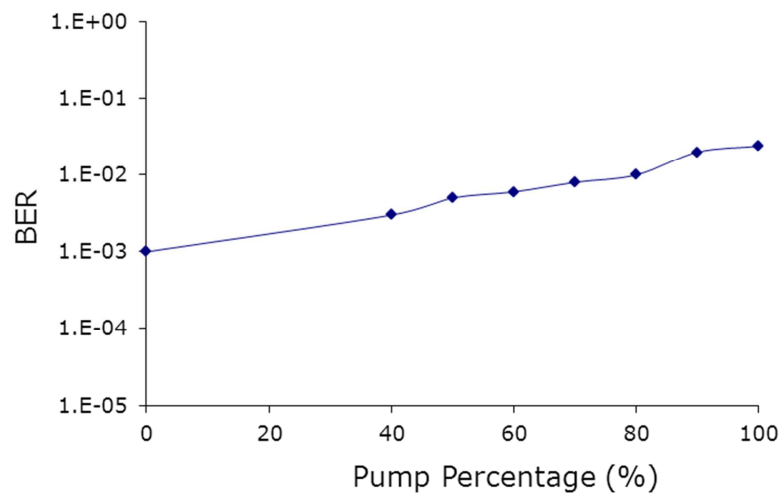

Figure 7. BER vs Raman pump.
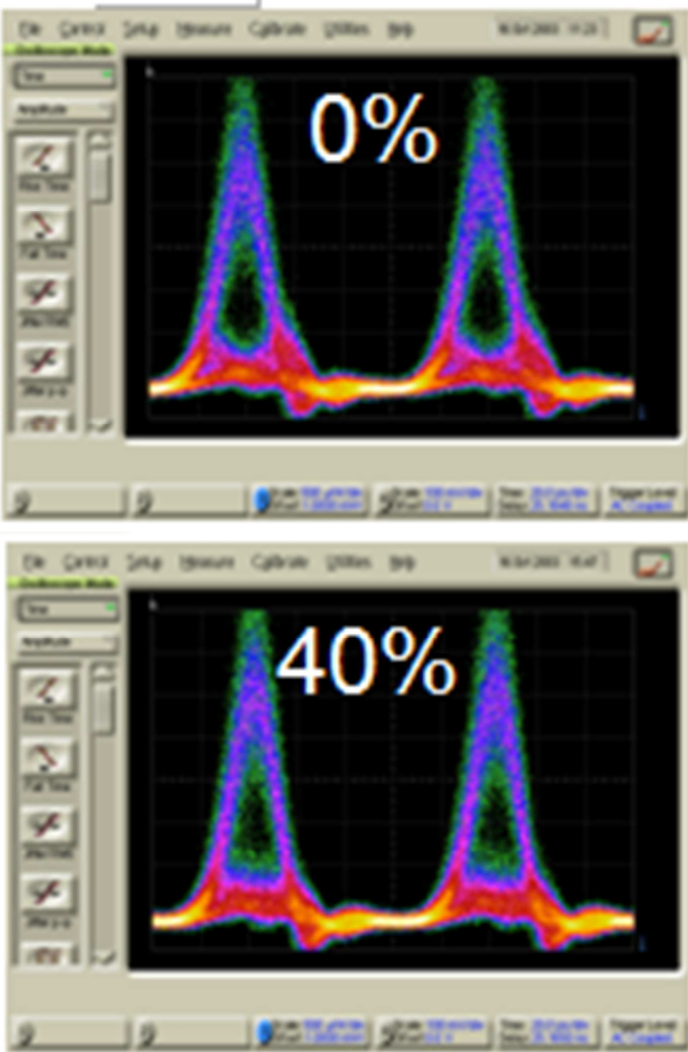
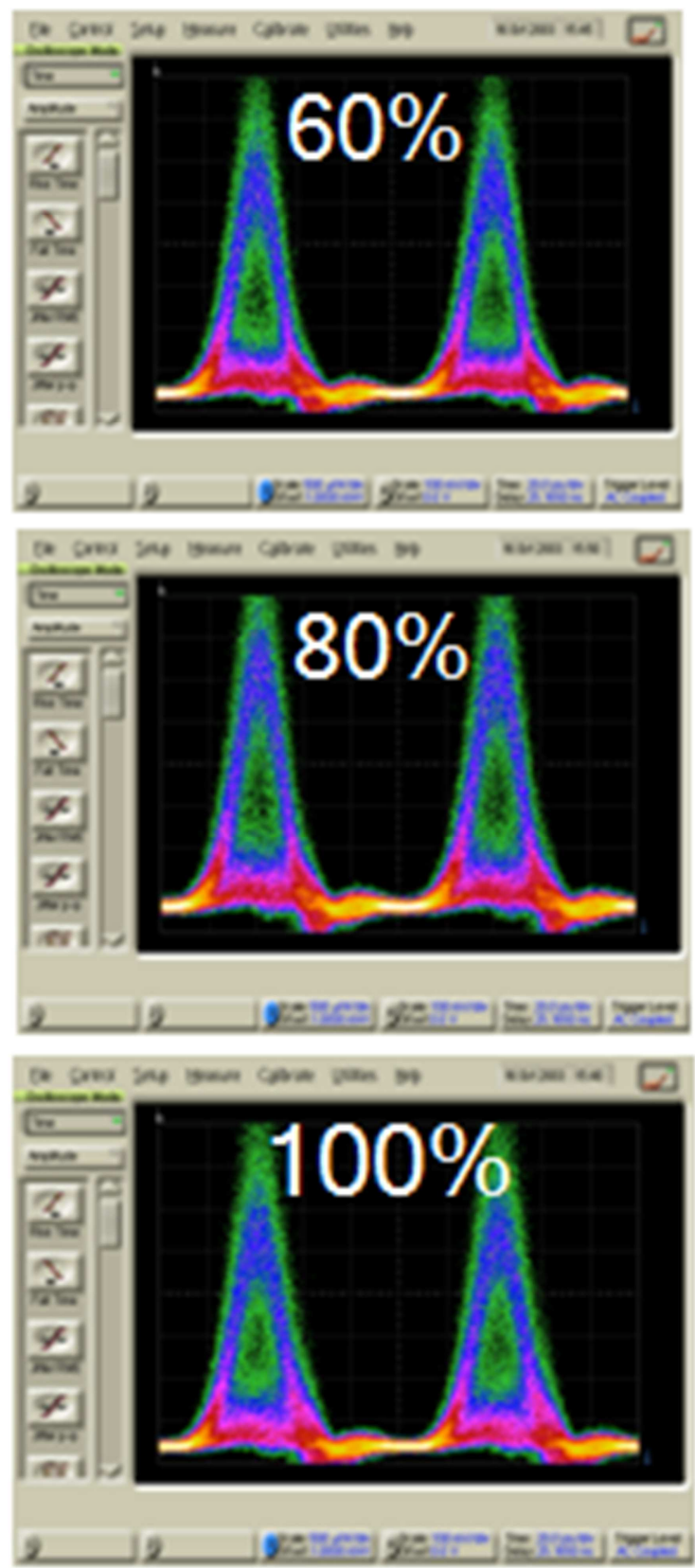

Figure 8. Received eye diagrams with different Raman pumps.

To solve the above problem, the lumped EDFA gains are empirically reduced such that the accumulated ASE noise is improved and the entire spectrum is nearly flattened. By doing this, all EDFA gains in our system (excluding the booster and pre-amplifier) are dropped by about $2 \mathrm{~dB}$ and no longer operate at saturation. This essential adjustment allows using higher Raman powers comfortably where it is noticed that the signal performance is now improved with Raman rather than degraded as before. In this case, the Raman amplification effectively compensates for the drops in the Erbium gain thus the data signal power throughout the system is balanced on average. As a result, successful transmission of the $40 \mathrm{~Gb} / \mathrm{s}$ data is achieved where the best signal is obtained by using the highest Raman percentage (100\%) which is corresponding to $1.58 \mathrm{~W}$. The eye diagram 
of the received $10 \mathrm{~Gb} / \mathrm{s}$ signal is presented in Fig. 9 where it measures $\sim 10^{-9}$ BER. We believe that this result is satisfactory for backward Raman amplification, and there is no need to afford higher Raman power as long as the intended OTDM data signal is successfully transmitted and received via our system.

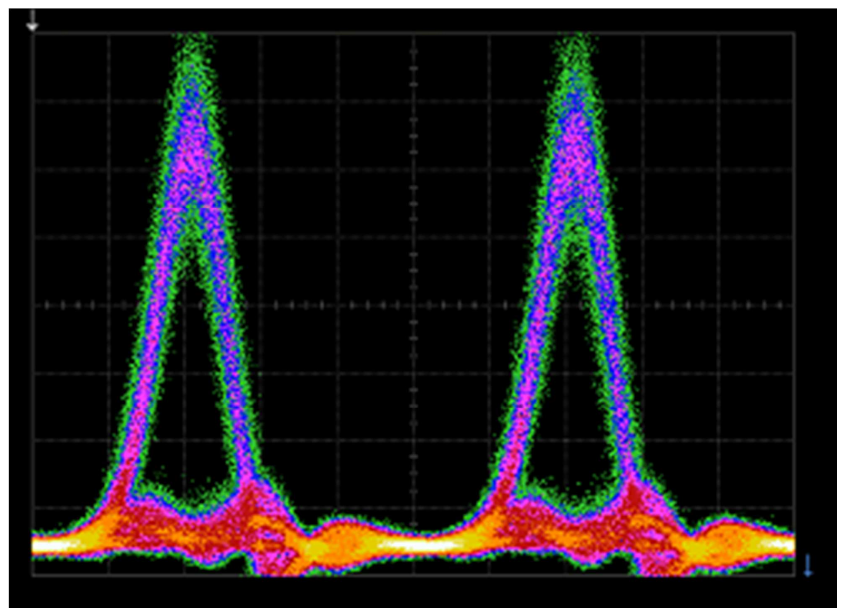

Figure 9. Received signal obtained by $1.58 \mathrm{~W}$ Raman pump and reduced Erbium gain.

In fact, this kind of results can encourage upgrading systems that use traditional fiber types/configurations where the above study can be expanded over larger systems such as long and ultra-long haul transmission systems, whether having real or recirculating-loop-based setup [19-20]. These large systems are composed of multiple unrepeatered fiber spans that are typically $<200 \mathrm{~km}$ and are conventionally amplified by EDFAs. To make use of the above results without major alteration on the basic configuration, it is possible to insert multiple Raman pumps where each one can serve single unrepeatered $200 \mathrm{~km}$ SMF span. In this case, each section of the entire system can be simulated by our single in-line long fiber span presented in this paper. The main purpose in such large systems would be reducing the required lumped Erbium gain (hence ASE noise) such that the total number of EDFAs can be reduced significantly while the overall performance is improved. Additional work can also be done where the investigation can be extended to be applied with complex modulation formats such as QPSK, DPSK, etc.

\section{Conclusions}

In this paper, we demonstrate unrepeatered $40 \mathrm{~Gb} / \mathrm{s}$ OTDM data transmission over $206 \mathrm{~km}$ conventional SMF span using backward Raman amplification. The experiment uses dispersion-managed SMF-DCF configuration so that Raman amplification is applied to compensate for the SMF span loss while remote EDFAs are used for the DCF spans. The system is optimized with respect to the operating wavelength, Erbium gains/spectra and Raman pump power. As a result, successful transmission of the intended OTDM signal is attained using $1545 \mathrm{~nm}$ signal wavelength, $1.58 \mathrm{~W}$
Raman power and unsaturated gains in the cascaded EDFAs.

\section{Acknowledgments}

The author thanks Aston Photonics Research Group and Azea Networks for their assistance in this experiment.

\section{References}

[1] R. W. Boyd, Nonlinear Optics, San Diego, 2008.

[2] G. P. Agrawal, Nonlinear Fiber Optics, San Diego, 2007.

[3] M. Islam, "Raman amplifiers for telecommunications", IEEE Journal of Selected Topics In Quantum Electronics, vol. 8, no. 3, pp. 548-559, 2002.

[4] Z. Xu, J. Seoane, A. Siahlo, L. Oxenlewe, A. Clausen, and P. Jeppesen, "Experimental characterization of dispersion maps with Raman gain in $160 \mathrm{~Gb} / \mathrm{s}$ transmission systems", Conference on Lasers and Electro-Optics (CLEO), pp. 2, 2004.

[5] M. Haris, J. Yu, and G. K. Chang, "Repeaterless transmission of $10 \mathrm{Gbit} / \mathrm{s}$ MD-RZ signal over $300 \mathrm{~km}$ SMF-28 by using Raman amplification", IEEE Lasers and Electro-Optics Society (LEOS), WE1, pp. 479-480, 2005.

[6] H. Maeda, G. Funatsu, and A. Naka, "Ultra-long-span 500 km $16 \times 10 \mathrm{Gbit} / \mathrm{s}$ WDM unrepeatered transmission using RZDPSK format", Electronics Letters, vol. 41, no. 1, pp. 34-35, 2005.

[7] R. Jee, and S. Chandra, "Single-span transmission of WDM RZ-DPSK signal over $310 \mathrm{~km}$ standard SMF without using FEC and remote-pumping", International Conference on Advances in Computing, Communications and Informatics (ICACCI), pp. 172-177, 2015.

[8] Z. Huang, A. Gray, Y. Lee, I. Khrushchev, and I. Bennion, "All-Raman amplified transmission at $40 \mathrm{Gbit} / \mathrm{s}$ in standard single mode fiber", Conference on Lasers and Electro-Optics (CLEO), pp. 531, 2003.

[9] G. Charlet, M. Salsi, P. Tran, M. Bertolini, H. Mardoyan, J. Renaudier, O. Bertran-Pardo, and S. Bigo, "72 × $100 \mathrm{~Gb} / \mathrm{s}$ transmission over transoceanic distance, using large effective area fiber, hybrid Raman-erbium amplification and coherent detection", Optical Fiber Communication Conference, p. PDPB6, 2009.

[10] D. Chang, W. Pelouch, P. Perrier, H. Fevrier, S. Ten, C. Towery, and S. Makovejs, "150 × $120 \mathrm{~Gb} / \mathrm{s}$ unrepeatered transmission over $409.6 \mathrm{~km}$ of large effective area fiber with commercial Raman DWDM system", Optics Express, vol. 22, no. 25, pp. 31057-31062, 2014.

[11] H. Bissessur, C. Bastide, S. Dubost, and S. Etienne, " $80 \times 200$ $\mathrm{Gb} / \mathrm{s}$ 16-QAM unrepeatered transmission over $321 \mathrm{~km}$ with third order Raman amplification", Optical Fiber Communications Conference and Exhibition (OFC), pp. 1-3, 2015.

[12] H. Masuda, H. Kawakami, S. Kuwahara, A. Hirano, K. Sato, and Y. Miyamoto, "1.28 Tbit/s $(32 \times 43 \mathrm{Gbit} / \mathrm{s})$ field trial over $528 \mathrm{~km}(6 \times 88 \mathrm{~km})$ DSF using L-band remotely-pumped EDF/distributed Raman hybrid inline amplifiers", Electronics Letters, vol. 39, no. 23, pp. 1668-1670, 2003. 
[13] D. Rafique, T. Rahman, A. Napoli, R. Palmer, J. Slovak, E. Man, S. Fedderwitz, M. Kuschnerov, U. Feiste, B. Spinnler, B. Sommerkorn-Krombholz, and M. Bohn, "9.6 Tb/s CP-QPSK transmission over $6500 \mathrm{~km}$ of NZ-DSF with commercial hybrid amplifiers", IEEE Photonics Technology Letters, vol. 27 , no. 18, pp. 1911-1914, 2015.

[14] P. Rosa, N. Murray, R. Bhamber, J. Ania-Castañón, and P. Harper, "Unrepeatered $8 \times 40 \mathrm{~Gb} / \mathrm{s}$ transmission over $320 \mathrm{~km}$ SMF-28 using ultra-long Raman fiber laser based amplification", European Conference on Optical Communications (ECOC), pp. 1-3, 2012.

[15] D. Chang, S. Burtsev, W. Pelouch, E. Zak, H. Pedro, W. Szeto, H. Fevrier, T. Xia, and G. Wellbrock, " $150 \times 120 \mathrm{~Gb} / \mathrm{s}$ field trial over 1,504 km using all-distributed Raman amplification", Optical Fiber Communications Conference and Exhibition (OFC), pp. 1-3, 2014.

[16] S. Burtsev, H. Perdo, D. Chang, W. Pelouch, H. Fevrier, S. Ten, S. Makovejs, and C. Towery, " $150 \times 120 \mathrm{~Gb} / \mathrm{s}$ transmission over $3,780 \mathrm{~km}$ of G.652 fiber using all- distributed Raman amplification", Optical Fiber Communications Conference and Exhibition (OFC), pp. 1-3, 2015.

[17] M. Gunkel, F. Kiippers, J. Berger, U. Feiste, R. Ludwig, C. Schubert, C. Schmidt, and H. Weber, "Unrepeatered $40 \mathrm{Gbit} / \mathrm{s}$ RZ transmission over $252 \mathrm{~km}$ SMF using Raman amplification”, Electronics Letters, vol. 37, no. 10, pp. 646648, 2001.

[18] M. Nahas, "Investigation of different techniques to upgrade legacy WDM communication systems", PhD Thesis, Aston University, 2006.

[19] M. Nahas, and K. Blow, "Monitoring long distance WDM communication lines using a high-loss loopback supervisory system", Optics Communications, vol. 285, no. 10, pp. 26202626, 2012.

[20] M. Nahas, and K. Blow, "Investigation of the effect of an increased supervisory signal power in a high-loss loopback monitoring system", IET Communications, vol. 8, no. 6, pp. 800-804, 2014. 